\title{
OBTENCIÓN DE BIODIESEL A PARTIR DE DIFERENTES TIPOS DE GRASA RESIDUAL DE ORIGEN ANIMAL
}

\author{
CANDELARIA TEJADA TOVAR ${ }^{1}$; LESLY TEJEDA BENÍTEZ ${ }^{2}$; \\ ÁNGEL VILLABONA ORTIZ ${ }^{3}$; LUIS MONROY RODRÍGUEZ ${ }^{4}$
}

\author{
Recibido el 6 de julio de 2012 y aprobado el 17 de enero de \\ 2013
}

\section{RESUMEN}

Se estudian diferentes posibilidades de obtención de biodiesel a partir de residuos grasos generados en la explotación pecuaria: grasa de pollo, cerdo, y vacuna, para ello se analizan las variables relación molar alcohol/grasa animal y cantidad de catalizador, puesto que son las que más inciden en la calidad del biodiesel, al evaluar sus características y rendimientos de la reacción de transesterificación.

El rendimiento en el proceso de extracción de la grasa de material de desecho de pollo es del 70,5\%, y de la grasa de cerdo del $90 \%$, por lo que se plantean como alternativas viables desde el punto de vista técnico, permitiendo la valorización de estos desechos orgánicos y aliviando la contaminación generada en este tipo de industrias. De la caracterización realizada a la grasa de pollo y cerdo se pudo establecer que ambas materias primas son de baja acidez lo cual garantiza un alto grado de transesterificación, teniendo en cuenta que tuvo un rendimiento del $96 \%$ a partir de la grasa de pollo y del $91,2 \%$ a partir de la grasa de cerdo. Del análisis cromatográfico del biodiesel sintetizado se obtuvo que el ácido graso más común fue el del ácido oleico.

\section{PALABRAS CLAVE}

Biodiesel, grasa residual, pecuario, rendimiento, transesterificación.

\section{EXTRACTING BIODIESEL FROM DIFFERENT TYPES OF ANIMAL ORIGIN RESIDUAL FAT}

\begin{abstract}
Different possibilities of extracting biodiesel from fat residues generated on farm livestock: chicken, pork, and bovine fat, were studied. To do this, the variables molar ratio alcohol/animal fat and amount of catalyst are analyzed, since those are the ones which most affect the quality of biodiesel when assessing the characteristics and efficiency of the transesterification reaction.
\end{abstract}

The efficiency in the poultry waste material extraction process is $70.5 \%$, and in pork fat is $90 \%$, reason why they are proposed as feasible alternatives from the technical standpoint, allowing the recovery of this organic waste, thus alleviating the pollution 
generated by this type of industry. From the characterization performed on chicken and pork fat, it has been established that this raw material has low acidity which ensures a high degree of transesterification, taking into account that it had 96\% efficiency from chicken fat and $91.2 \%$ from pork fat. From the synthesized biodiesel Chromatographic analysis it was found that the most common fatty acid was oleic acid.

\section{KEY WORDS}

Biodiesel, residual fat, livestock, effficiency, transesterification.

\section{MARCO CONCEPTUAL}

\section{Biodiesel: definiciones y características}

El biodiesel se define como un metil éster obtenido a partir de aceites vegetales o grasas animales, de características similares al diesel, que se prestan a sustituirlo total o parcialmente como combustible de motores de combustión interna, puesto que su uso representa una excelente opción desde el punto de vista ecológico al reducir las emisiones de dióxido de carbono y la dependencia de fuentes de energía no renovables (Encinar et al., 2011).

Generalmente las personas Ilaman "biodiesel" a cualquier combustible para motores diesel que sea originado de vegetales o animales, como el aceite usado para freír, sin cualquier tratamiento. Sin embargo, las definiciones de "biodiesel" que poseen mayor consistencia técnica son aquellas que caracterizan su composición química (mono-alquil ésteres de ácidos grasos), su origen (vegetal, animal), su empleo y sus características técnicas.

La mayoría del biodiesel producido en el mundo es proveniente de aceites vegetales principalmente del aceite de colza en Europa y Canadá, y aceite de soya en Estados Unidos (Andersen \& Weinbach, 2010). En Colombia, la principal materia prima para la producción de biodiesel es el aceite de palma, pues actualmente cuenta con más de 300.000 hectáreas sembradas en palma de aceite y cinco plantas productoras de biodiesel (Federación Nacional de Biocombustibles de Colombia, 2012).

Es destacable la obtención de biodiesel a partir de materias primas alternativas, como los residuos grasos animales y aceites de fritura usado, utilizadas principalmente en países como Canadá, México e Irlanda (Atabani et al., 2012), estas son una materia prima ideal a bajo costo, ya que normalmente el biodiesel se obtiene de aceites vegetales de alto valor que representan cerca del $85 \%$ de los costos de producción. Cabe mencionar que las grasas animales son altamente viscosas y en su mayoría sólida a temperatura ambiente, debido a su alto 
contenido de ácidos grasos saturados, por lo que su uso como combustibles puede conducir a mala atomización del mismo, y en consecuencia una combustión incompleta. La transesterificación y la emulsificación son dos de las soluciones principales que han aparecido como métodos eficaces para el uso de grasas animales en el motor diesel. Además, su índice de cetano alto y los valores de calefacción están cerca del combustible diesel, al igual que su contenido de oxígeno, que mejora la combustión con menor relación aire/combustible, así mismo se ha demostrado que existe una buena disponibilidad de estas materias primas, evitando los debates éticos de utilizar cultivos y tierras que se destinan para fines alimenticios, además estimaciones hechas en Noruega por Andersen y Weinbach (2010) muestran que es posible obtener 2,5 toneladas de ácidos grasos de residuos de origen animal por cada 1000 familias al año (Basha, Gopal \& Jebaraj, 2009; Öner \& Altun, 2009; Rivera, Villanueva \& Sandoval, 2009; Encinar et al., 2011).

El biodiesel tiene en general un poder calórico inferior algo menor al diesel $(7,795 \mathrm{kcal} / \mathrm{l}$ vs. 8,74 kcla/l). Su viscosidad cinemática en general está entre 1,9 y $6,0 \mathrm{cSt}$, aunque este parámetro no difiere sustancialmente del gasoil (1,3-4,1 cSt). Su densidad es de aproximadamente $0,878 \mathrm{~kg} / \mathrm{L}$ a $15^{\circ} \mathrm{C}$, y su flash point llega a sobrepasar los $130^{\circ} \mathrm{C}$, a diferencia del gasoil, cuyo punto de inflamación es de apenas $60-80^{\circ} \mathrm{C}$, lo que lo hace más seguro como combustible. Posee además un número cetano ligeramente mayor al del gasoil, y duplica el poder de lubricación del mismo.

En la Tabla 1 se muestra un resumen de las características típicas del biodiesel y del diesel petrolífero.

Tabla 1. Propiedades del biodiesel y el diesel

\begin{tabular}{|ccc|}
\hline Datos fisico-químicos & Biodiesel & Diesel \\
Composición combustible & Ester metílico & Hidrocarburo \\
Poder calorifico inferior, kcal/kg (aprox.) & Acidos grasos C12-C22 & $10-\mathrm{C} 21$ \\
Viscosidad cinemática, cSt (a 40 ${ }^{\circ} \mathrm{C}$ ) & 9500 & 10800 \\
Peso específico, $\mathrm{g} / \mathrm{cm}^{3}$ & $3,5-5,0$ & $3,0-4,5$ \\
Azufre, $\% \mathrm{P}$ & $0,875-0,900$ & 0,850 \\
Punto ebullición, ${ }^{\circ} \mathrm{C}$ & 0 & 0,2 \\
Punto inflamación, ${ }^{\circ} \mathrm{C}$ & $190-340$ & $180-335$ \\
Punto escurrimiento, ${ }^{\circ} \mathrm{C}$ & $120-170$ & $60-80$ \\
Número cetano & $-15 /+16$ & $-35 /-15$ \\
Relación estequiométrica Aire/comb. $\mathrm{p} / \mathrm{p}$ & $48-60$ & 46 \\
\hline Fuente: Larosa (2003). & 13,8 & 15 \\
\hline
\end{tabular}

De la Tabla 1, se aprecia que el biodiesel presenta excelentes propiedades en cuanto a favorecer la adecuada combustión, tales como el número de cetano, que es más alto que en el diesel de petróleo retrasando la autoignición del combustible al inyectarse al motor, igual sucede con el punto de inflamación, que reduce el peligro de incendio durante su manejo, sin 
embargo, su poder calorífico y punto de escurrimiento son menores, presentando por tanto un mayor consumo de combustible y posibles problemas de fluidez en lugares fríos (Awad et al., 2012).

\section{Tecnología para la producción de biodiesel}

La obtención de biodiesel se realiza principalmente mediante: microemulsión, pirólisis, dilución y transesterificación, esta última es la vía más empleada para producirlo, ya que es la más económica, ofreciendo ventajas como: elevada conversión (98\%) con pocas reacciones secundarias y reducido tiempo de reacción y conversión directa a éster sin pasos intermedios (Lin et al., 2011).

La transesterificación de grasas y aceites, comprende la reacción de estas con alcohol metílico en ambiente básico, una molécula de triglicéridos, componente mayoritario en una grasa, reacciona con un alcohol primario, bajo la acción de un catalizador, para producir una mezcla de ésteres de ácidos grasos y glicerina, esta consta de tres etapas consecutivas en las que el triglicérido es convertido en diglicéridos, monoglicéridos y glicerina (Arévalo, Ulloa \& Astudillo, 2008; Math, Kumar \& Chetty, 2010; Keera, El Sabagh \& Taman, 2011).

Tal como se muestra en las siguientes reacciones:

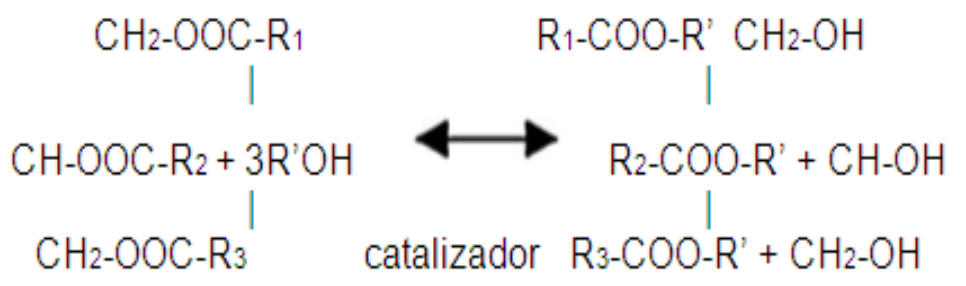

Fuente: Shahid y Jamal (2011).

Los catalizadores a emplear pueden ser soda cáustica o metilato sódico, ambos en solución metanólica. La tecnología básica de producción consta de un reactor en el cual se lleva a cabo la transesterificación. Por un lado, en un tanque auxiliar, se prepara la solución de metóxido de sodio, a partir de alcohol -usualmente metílico- de alta pureza (el agua interfiere en la reacción) e hidróxido de sodio. No se utiliza alcóxido anhidro pues la reacción de dicha sustancia en agua es muy violenta y tiende a la autoignición. Esta mezcla fuertemente cáustica es vertida en el reactor principal que contiene los lípidos fundidos. La reacción a menudo se realiza a unos $50^{\circ} \mathrm{C}$ para agilizar la misma. En los procesos industriales más complejos, se procede a separar el biodiesel y la glicerina formada mediante el uso de centrífugas que permiten remover constantemente los productos de reacción. Sin embargo, aún son comunes los procesos por lotes, en los que se procede a una decantación de la glicerina. Luego de separar la misma, el éster es lavado para 
eliminar jabones y otros subproductos de reacción indeseados (Larosa, 2003).

\section{Ventajas y desventajas del biodiesel}

La obtención y uso del biodiesel trae ventajas innumerables, así como desventajas, entre las que se destacan las siguientes:

\section{Ventajas}

No requiere mayores modificaciones para su uso en motores diesel comunes. Permite al productor agropecuario autoabastecerse de combustible y a países agrícolas independizarse de los países productores de petróleo. Tiene un gran poder de lubricación y minimiza el desgaste del motor. Se ha mostrado por muchos estudios como una alternativa de combustible que reduce las emisiones de: monóxido de carbono (CO), hidrocarburos $(\mathrm{HC})$, dióxido de azufre $\left(\mathrm{SO}_{2}\right)$, hidrocarburos aromáticos policíclicos (HAP), y material particulado (PM), en comparación con el combustible diesel. Su rendimiento en motores es similar al diesel común. Puede utilizarse en mezclas con gasoil común en cualquier proporción. Reduce en gran medida los humos visibles durante el arranque. Posee una gran biodegradabilidad. Es aproximadamente diez veces menos tóxico que la sal común de mesa. Su transporte y almacenamiento es más seguro dado su alto flash point. No contiene azufre, y permite el uso de catalizadores. No es nocivo para la salud humana, para la vegetación, los animales vivos y no daña monumentos y/o edificios. El olor de combustión asemeja el olor a fritura, a diferencia del olor del gasoil (Lenoir, 2002; Öner \& Altun, 2009; Balat \& Balat, 2010).

\section{Desventajas}

Presenta elevados costos de materia prima, aunque en el caso de Colombia por ser un país agrícola y pecuario no habría problema. Su combustión puede acarrear un aumento de óxidos de nitrógeno (NOX). Presenta problemas de fluidez a bajas temperaturas (menores a $0^{\circ} \mathrm{C}$ ). Presenta escasa estabilidad oxidativa, y su almacenamiento no es aconsejable por períodos superiores a seis meses. Su poder solvente lo hace incompatible con una serie de plásticos y elementos derivados del caucho natural, y a veces obliga a sustituir mangueras en el motor. Su carga en tanques ya sucios por depósitos provenientes del gasoil puede presentar problemas cuando por su poder solvente "limpia" dichos depósitos (Lenoir, 2002; Balat \& Balat, 2010).

\section{METODOLOGÍA}

Teniendo en cuenta el procedimiento general para la obtención de biodiesel descrito en el marco teórico, algunas de las variables que inciden en la calidad de este son: la pureza de los 
reactivos, la relación molar alcohol/grasa animal, tipo y cantidad de catalizador, agitación, temperatura y tiempo de reacción. Sin embargo, solo se tomaron las variables: relación molar alcohol/grasa animal, y cantidad de catalizador, como factores para el diseño de experimentos.

\section{Selección de variables}

Selección de materias primas: En el presente trabajo fueron seleccionadas como materias primas, grasas de origen animal, consideradas como subproductos originados de la actividad pecuaria que no son utilizados para consumo humano: grasa de pollo, grasa vacuna, y grasa de cerdo.

Catalizador: Los catalizadores que se emplean comúnmente en el proceso de transesterificación son básicos tales como el metóxido e hidróxido de sodio y potasio (Barrera, 2006), estos son catalizadores homogéneos y se escogieron debido a que la temperatura, tiempo de reacción, y cantidad de alcohol son menores al compararlos con otro tipo de catalizador, razón por la cual se decidió trabajar con hidróxido de sodio y potasio. Lo anterior aplica para trabajar con grasas de origen animal a excepción de la grasa vacuna en la cual se debe anteceder una catálisis ácida con ácido sulfúrico, con el fin de transformar los ácidos grasos como los triglicéridos (Fredman, Butterfield \& Pryde, 1986).

Tipo de alcohol: Según la CEPAL y Naciones Unidas (2007), el proceso de producción de biodiesel necesita de un alcohol para la reacción. Los alcoholes por excelencia para la producción de biodiesel son el metanol y el etanol, en particular el metanol por sus propiedades físico-químicas, reacciona más rápidamente con los triglicéridos y en la mayoría de los países es más económico que el etanol. En general desde el punto de vista técnico de la reacción, el metanol presenta ventajas: requiere menor cantidad de alcohol en exceso que el etanol; posee mayor eficiencia de conversión de aceites; requiere menores temperaturas de reacción; y posee menor tiempo de reacción, por estas razones, se decidió usar metanol en el proceso.

Tiempo de reacción: Darnoko y Cheryan (2000), y Keera, El Sabagh y Taman (2011), encontraron que la reacción de transesterificación es muy rápida, y cerca del $80 \%$ de la conversión tiene lugar en los primeros 30 minutos, después de la primera hora se alcanza entre el 93 y el $98 \%$ de conversión, se obtiene el mejor rendimiento luego de hora y media, por lo cual se tomó este valor fijo.

Temperatura de reacción: Según Darnoko y Cheryan (2000) la temperatura para llevar a cabo la obtención de biodiesel es de $60^{\circ} \mathrm{C}$, teniendo en cuenta que el punto de ebullición del metanol es $64^{\circ} \mathrm{C}$. Por lo cual se tomó también como un valor fijo.

Agitación: Según lo reportado por Darnoko y Cheryan (2000) es adecuado un valor entre 120-600 rpm, se tomó de $300 \mathrm{rpm}$. 
Rendimiento de la reacción: Para la presente investigación el rendimiento de la reacción es la variable respuesta, expresada como la relación entre la masa de biodiesel obtenido y la masa de grasa animal utilizada.

\section{Diseño de experimentos}

Se empleó un modelo factorial tipo $1^{k}$ en donde $K$ son los factores y el uno representa los niveles de cada factor, en este caso $\mathrm{K}$ es 2 (cantidad de catalizador y relación aceite/grasa animal), las restricciones del sistema son: temperatura, velocidad de agitación, tiempo de reacción y presión, para los cuales se asumieron valores fijos.

Los niveles de cada factor se toman dentro de los rangos estipulados en la literatura. Darnoko y Cheryan (2000), y Keera, El Sabagh y Taman (2011), reportan para el catalizador un rango entre $0,5 \%$ y $2 \%$, considerando el valor óptimo de 1 , puesto que el rendimiento decrece y se favorece la saponificación usando valores mayores a este, por lo cual se tomó como un valor fijo. De igual forma, para la relación metanol/grasa animal, se reporta que una relación 6:1 se considera adecuada (Arbeláez \& Rivera, 2007), por lo cual también se tomó como un valor fijo, aunque por estequiometría de la reacción se requiere una relación molar alcohol/grasa de 3:1 para convertir los triglicéridos a alquilésteres, como la reacción es reversible, se agrega un exceso de alcohol para favorecer la formación de ésteres (Rojas \& Girón, 2011).

Tabla 2. Diseño de experimentos

\begin{tabular}{|lcc|}
\hline Materia prima & $\begin{array}{c}\text { Relación metanol/grasa } \\
\text { (mol/mol) }\end{array}$ & $\begin{array}{c}\text { Cantidad de catalizador } \\
(\% \text { peso })\end{array}$ \\
Grasa vacuna & $6: 1$ & 1 \\
Grasa de cerdo & $6: 1$ & 1 \\
Grasa de pollo & $6: 1$ & 1 \\
\hline Fuente: Elaborada por los autores de acuerdo a la bibliografia consultada.
\end{tabular}

\section{Extracción de grasas de origen animal}

\section{Grasa de pollo}

Se seleccionaron 2000 gramos de material de desecho (pellejos y sebo), sometiéndolos inicialmente a proceso de lavado y escurrido para eliminar impurezas, seguidamente se llevó a fundición en una mufla a $75^{\circ} \mathrm{C}$ por el término de una hora y media, obteniéndose 1408,2 gramos de grasa líquida para un rendimiento en la extracción del $70,5 \%$. Seguidamente, la grasa obtenida fue llevada a un proceso de winterizado, que consistió en enfriar la grasa obtenida a $7^{\circ} \mathrm{C}$ durante 8 horas, con la finalidad de separar por precipitado las gomas y mucílagos con lo cual se logra mejorar su apariencia lográndose un color amarillo intenso y transparente, el rendimiento en este proceso fue del $85 \%$. 


\section{Grasa de cerdo}

La primera etapa del proceso consistió en la selección de las vísceras de cerdo para obtener una mayor cantidad de grasa. Luego se pesaron y lavaron 2000 gramos, utilizando para ello únicamente agua potable. Los residuos se colocaron en un recipiente metálico y se llevaron directamente a una estufa, hasta fundir toda la grasa, manteniendo la temperatura constante en $60^{\circ} \mathrm{C}$, con agitación periódica, para agilizar el proceso y obtener mejores resultados. Al cabo de 1,75 horas se obtuvieron $1800 \mathrm{ml}$, al finalizar el proceso se tomaron los residuos y se pesaron 300 gramos, para un rendimiento del $90 \%$ en el proceso de extracción de la grasa.

\section{Grasa vacuna}

La grasa vacuna fue conseguida en un establecimiento local (carnicería). Para la extracción de la grasa se cortó el sebo en pedazos muy pequeños, posteriormente se sometió a un proceso de pirólisis a una temperatura que oscila entre los $100^{\circ} \mathrm{C}$ y $180^{\circ} \mathrm{C}$, estabilizándose a los $140^{\circ} \mathrm{C}$ por un tiempo estimado de 3 horas; con el fin de obtener la grasa vacuna por fundición.

Fraccionamiento a $50^{\circ} \mathrm{C}$ : La grasa vacuna fue estabilizada a $50^{\circ} \mathrm{C}$ durante una hora en un sistema con agitación controlada (agitador mecánico). Luego se disminuyó la temperatura a una velocidad de $0,033^{\circ} \mathrm{C} / \mathrm{min}$ hasta $45^{\circ} \mathrm{C}$, temperatura a la cual se estabilizó durante una hora. Posteriormente por filtración a vacío en un embudo con camisa termostatizada a $45^{\circ} \mathrm{C}$, fueron separadas la oleína y la estearina. Así mismo, se continuó trabajando durante las horas siguientes, bajando la temperatura cada 5 minutos hasta llegar a $40^{\circ} \mathrm{C}$, que es la temperatura mínima que indica formación de cristales en la grasa.

A diferencia de la grasa de cerdo y la de pollo, la grasa vacuna se solidifica a temperatura ambiente, como alternativa a esta situación, Veira, Grompone y Jachmanián (2004), recomiendan una destilación fraccionada; tratando de buscar una solución a la solidificación de la grasa vacuna. En el presente estudio no se obtuvieron buenos resultados, por lo cual solo se obtuvo biodiesel a partir de la grasa de pollo y cerdo.

\section{Proceso de obtención del biodiesel}

El procedimiento para la obtención de biodiesel, incluyó los siguientes pasos: preparación del metóxido, reacción de transesterificación, separación del biodiesel y la glicerina por decantación, lavado del biodiesel y secado.

\section{Biodiesel a partir de grasa de pollo}

Se utilizaron como reactivos hidróxido de sodio como catalizador, y metanol. Luego de obtener la grasa, se utilizaron $50 \mathrm{ml}$ de grasa de pollo, $0,5 \mathrm{~g}$ de $\mathrm{NaOH}$ y $30 \mathrm{ml}$ de metanol; con 
agitación de 300rpm durante una hora y media, posteriormente la mezcla se transvasó a un embudo de decantación para separar el biodiesel y glicerina, como se observa en la Figura 1.

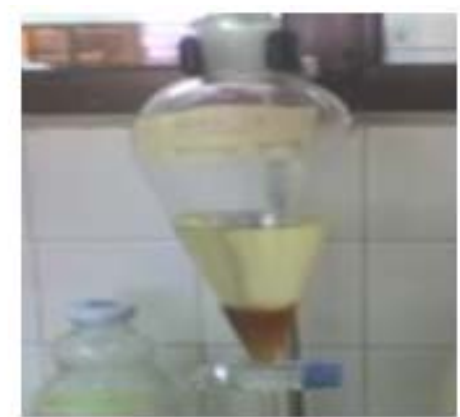

Figura 1. Biodiesel y glicerina obtenida de la grasa de pollo.

\section{Biodiesel a partir de grasa de cerdo}

Luego de obtener la grasa de cerdo, se utilizaron $125 \mathrm{ml}$ de grasa, 1 gramo de hidróxido de potasio y $24 \mathrm{ml}$ de metanol; con agitación a 300rpm durante 1 hora y media, luego de esto se transvasó a un embudo de decantación para separar el biodiesel de la glicerina (ver Figura 2).

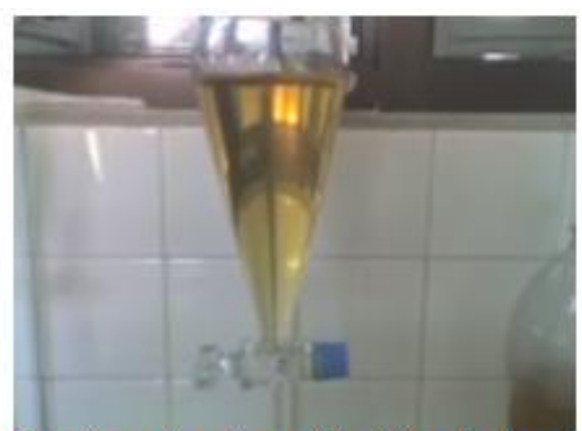

Figura 2. Biodiesel y glicerina obtenida de la grasa de cerdo.

\section{Biodiesel a partir de grasa de res}

Después del fraccionamiento se tomó una muestra de la grasa y se añadió metanol llevándose a cabo la catálisis ácida con ácido sulfúrico $\left(\mathrm{H}_{2} \mathrm{SO}_{4}\right)$ y la catálisis básica con hidróxido de potasio $(\mathrm{KOH})$, luego se efectúo el proceso de transesterificación por medio de un agitador magnético.

Se produjo la solidificación del producto obtenido por lo que no se obtuvieron los resultados esperados. Además, como lo reporta Vivas (2010), el problema más desfavorable y por el cual se restringe el uso de biodiesel bovino es la densidad $\left(0,8652 \mathrm{~g} / \mathrm{cm}^{3}\right.$ a $23^{\circ} \mathrm{C}$ ), ya que la norma (ASTM D4052) exige que se debe medir a $15^{\circ} \mathrm{C}$, temperatura a la cual el metil éster se solidifica. 


\section{Caracterización del biodiesel obtenido}

Para la evaluación de la calidad del biodiesel se determinaron propiedades como: densidad, punto de ebullición e inflamabilidad según la norma ASTM para calidad de biodiesel. ASTM ha especificado distintas pruebas que se deben realizar a los combustibles para asegurar su correcto funcionamiento, tomando esto como referencia, la densidad se evaluó de acuerdo a la norma en ISO 3675 a $15^{\circ} \mathrm{C}$ y para la viscosidad en ISO 3104 a $40^{\circ} \mathrm{C}$. Adicionalmente se realizó unos análisis por cromatografía a una muestra de los biodiesel obtenidos para analizar el contenido de ácidos grasos.

\section{RESULTADOS}

\section{Caracterización de materias primas}

El rendimiento en el proceso de extracción de la grasa de material de desecho del pollo es del 70,5\%, y de la grasa de cerdo es del $90 \%$, por lo que se plantean como alternativas económicamente rentables y viables desde el punto de vista técnico, permitiendo la valorización de estos desechos orgánicos, aliviando así la contaminación generada en la industria del pollo y porcina. Los resultados de la caracterización de las grasas animales se muestran en la Tabla 3.

Tabla 3. Caracterización de materias primas

\begin{tabular}{|lcccc|}
\hline Materia prima & $\begin{array}{c}\text { Densidad } \\
(\mathbf{g} / \mathbf{m l})\end{array}$ & $\begin{array}{c}\text { Acidez } \\
(\mathbf{m g ~ K O H} / \mathrm{g})\end{array}$ & $\begin{array}{c}\text { Indice de yodo } \\
(\mathbf{g} \text { de yodo/100 } \mathrm{g})\end{array}$ & $\begin{array}{c}\text { Indice de peróxido } \\
\text { (meq de peróxido } / \mathrm{kg})\end{array}$ \\
Grasa vacuna & 0,920 & 3,06 & 33,01 & 2,61 \\
Grasa de cerdo & 0,886 & 1,13 & 128,25 & 3,16 \\
Grasa de pollo & 0,876 & 2,77 & 47,92 & 14,27 \\
\hline
\end{tabular}

Fuente: Elaborada por los autores.

De la caracterización realizada a las materias primas, se pudo establecer que son de baja acidez lo que permitió experimentalmente obtener buenos resultados en la reacción de transesterificación, puesto que al usar un catalizador básico con materias primas de baja acidez se reduce la formación de jabones, y la saponificación del catalizador que puede tener lugar paralelamente a la transesterificación.

Por otro lado, la densidad de todas las grasas se encuentra dentro de los estándares, confirmando que es menor que la del agua, lo cual favorece los procesos de separación, referente al índice de yodo, un alto índice mejora las características del biodiesel obtenido y a su vez la estabilidad del biodiesel referida a su viscosidad. En cuanto al índice de peróxidos, el menor valor se presentó en la grasa vacuna, lo cual implica mayor estabilidad oxidativa, y por tanto es más favorable para la actividad del catalizador. 


\section{Caracterización de biodiesel de cerdo y pollo}

En este artículo la caracterización del biodiesel de origen pecuario se logró por medio de la técnica de cromatografía de gases de dos muestras, grasa de pollo y cerdo con metanol a una relación 6:1 alcohol/grasa, estos cromatogramas se muestran a continuación:

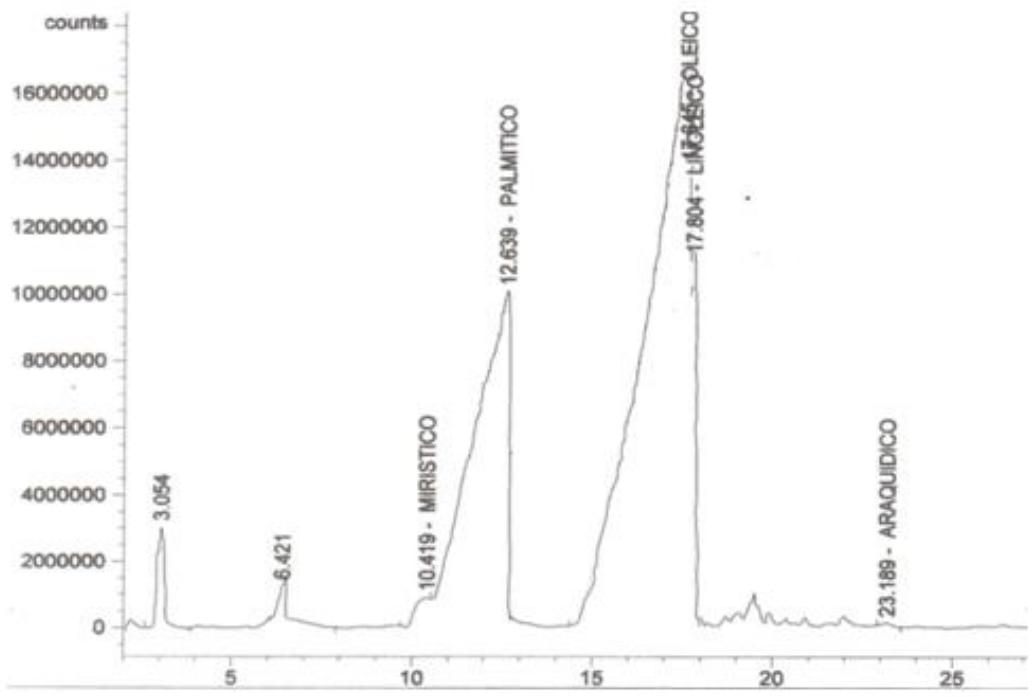

Figura 3. Cromatograma de gases de biodiesel de pollo.

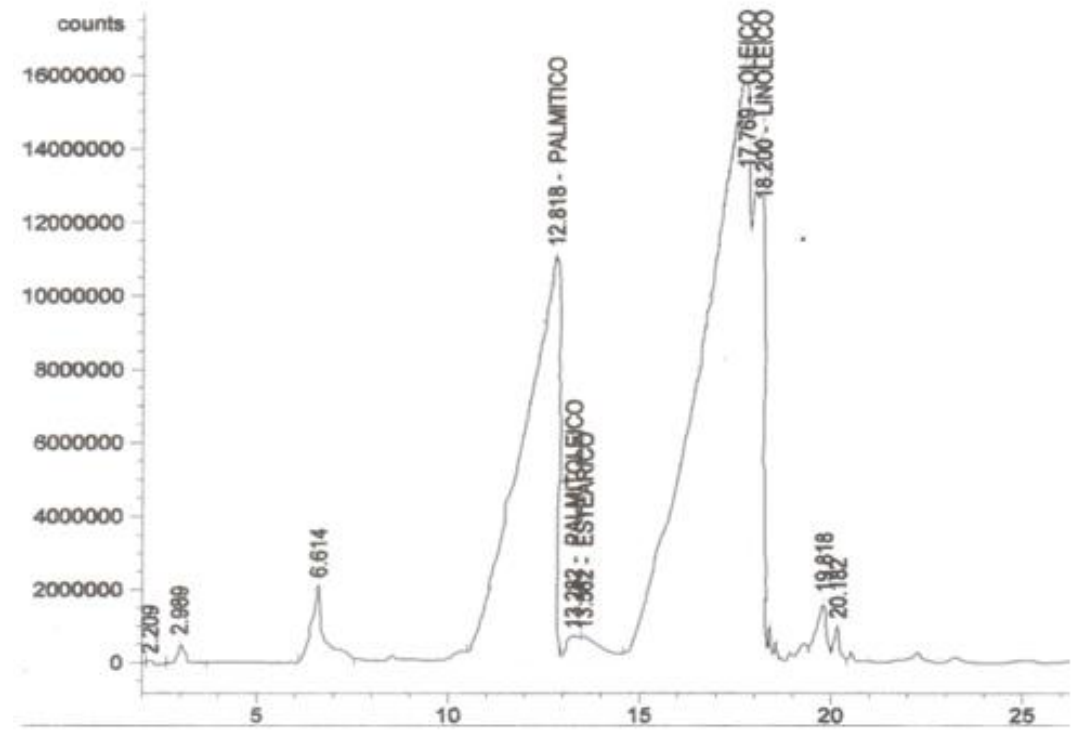

Figura 4. Cromatograma de gases de biodiesel de cerdo.

En la Figura 5 se resume un estudio comparativo entre los cromatogramas del biodiesel obtenido a partir de cada una de las materias primas, para seleccionar la mejor alternativa. 


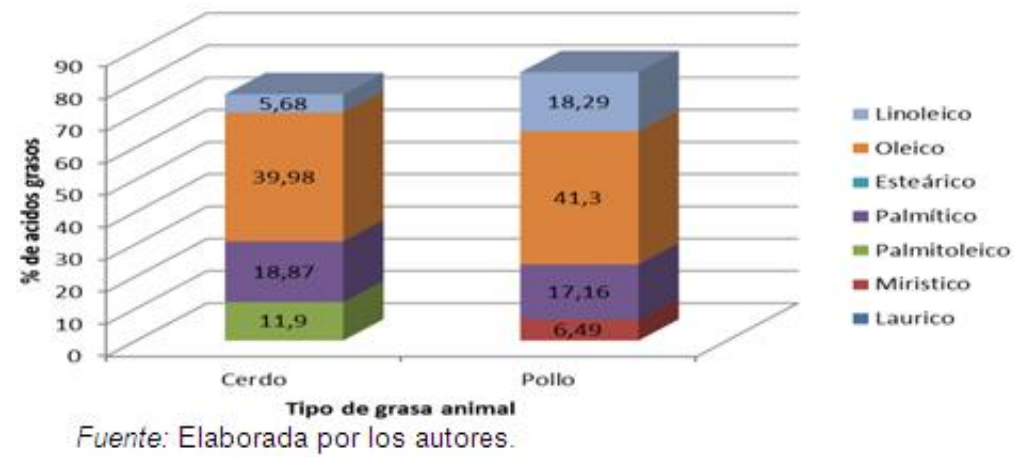

Figura 5. Comparación de ácidos grasos en los biodiesel obtenidos.

De lo anterior se deduce que la mayoría de ácidos grasos presentes en el biodiesel son insaturados, y en ambos es el ácido oleico el de mayor contribución, por lo que se espera un número de cetano bajo, además de una menor estabilidad produciendo residuos durante las reacciones de oxidación que se depositan en inyectores, lo que se traduce en una combustión incompleta, aunque no presentarán problemas de fluidez a bajas temperaturas. La Tabla 4 muestra los resultados de la caracterización realizada al biodiesel obtenido a partir de cada una de las materias primas estudiadas.

Tabla 4. Caracterización del biodiesel obtenido

\begin{tabular}{|lccccc|}
\hline Materia prima & $\begin{array}{c}\text { Densidad } \\
\text { relativa }\end{array}$ & $\begin{array}{c}\text { Punto de } \\
\text { inflamación } \\
\left({ }^{\circ} \mathbf{C}\right)\end{array}$ & $\begin{array}{c}\text { Viscosidad } \\
\text { cSt a } 40^{\circ} \mathrm{C}\end{array}$ & Inflamabilidad & $\begin{array}{c}\text { Rendimiento } \\
(\%)\end{array}$ \\
Grasa de pollo & 0,93 & 355 & 4,26 & Positiva & 96 \\
Grasa de cerdo & 0,93 & 360 & 3,48 & Positiva & 91,2 \\
\hline
\end{tabular}

Fuente: Elaborada por los autores

De la caracterización realizada a la grasa de pollo y cerdo se pudo establecer que ambas materias primas son de baja acidez lo que garantizaría un alto grado de transesterificación, esto se pudo comprobar en el proceso de obtención de biodiesel, teniendo en cuenta que el proceso de transesterificación tuvo un rendimiento del $96 \%$ a partir de la grasa de pollo y del $91,2 \%$ a partir de la grasa de cerdo, lo que se considera un buen rendimiento desde el punto de vista técnico y económico. Además, se establece que los parámetros medidos se encuentran dentro de las especificaciones, aunque de acuerdo a la norma EN 14241:2003, la densidad del biodiesel debe ser inferior a 0,90, sin embargo el punto de inflamación es alto, lo que reduce el peligro de incendio durante el uso o almacenamiento del mismo, por tanto ambos biodiesel son viables para su uso como combustible. La viscosidad cinemática del biodiesel es entre 10 y 15 veces más grande que el diesel, debido a que su masa molecular y estructura química es mayor. Altas viscosidades pueden afectar el volumen de flujo y la inyección en el motor, en general a bajas temperaturas compromete la integridad mecánica del sistema. El máximo permitido según ASTM D445 está entre 1,9-6,0 $\mathrm{mm}^{2} / \mathrm{s}$ y 3,5-5,0 
$\mathrm{mm}^{2} / \mathrm{s}$ en EN ISO 3104, por tanto ambos biodiesel obtenidos se encuentran dentro de las normas.

\section{CONCLUSIONES}

El biodiesel representa una importante fuente alternativa de energía ya que es un combustible renovable que puede ser obtenido de gran variedad de especies vegetales oleaginosas y grasas animales. Resulta menos perjudicial para el medio ambiente al no generar los mismos niveles de gases tóxicos que ocasionan el efecto invernadero. Además, la glicerina, como subproducto obtenido, puede ser aprovechada en aplicaciones como detergentes, aditivos alimentarios, productos cosméticos, lubricantes, entre otras.

El rendimiento en el proceso de extracción de la grasa de material de desecho del pollo es del 70,5\%, y de la grasa de cerdo es del $90 \%$, con lo que se plantean como alternativas viables para la obtención de biodiesel en Colombia, y sostenibles desde el punto de vista técnico, económico y ambiental permitiendo la valorización de los desechos orgánicos producidos por la industria pecuaria. De la caracterización realizada a la grasa de pollo y cerdo se pudo establecer que ambas materias primas son de baja acidez lo que garantizó un alto grado de transesterificación, al evitar la saponificación del catalizador.

De la caracterización realizada a las grasas de pollo y cerdo se pudo establecer que ambas son de baja acidez, esto garantizó un alto grado de transesterificación, lo que se pudo comprobar en el proceso de obtención de biodiesel, teniendo en cuenta que el proceso de transesterificación tuvo un rendimiento alto, además los parámetros medidos de los biodiesel obtenidos: densidad, viscosidad, y punto de inflamación, se encuentran dentro de las especificaciones.

\section{RECOMENDACIONES}

Se recomienda experimentar con diferentes tipos de catálisis, por ejemplo catálisis ácida o con lipasa, para tratar de resolver los inconvenientes presentados en la presente investigación con la grasa de res. 


\section{BIBLIOGRAFÍA}

- Andersen, O. y Weinbach, J.E. (2010). Residual animal fat and fish for biodiesel production. Potentials in Norway. Biomass and Bioenergy, 34(8), 1183-1188.

- Arbeláez, A. y Rivera, M. (2007). Diseño conceptual de un proceso para la producción de Biodiesel a partir de algunos aceites vegetales colombianos. Medellín: Escuela de Ingeniería Universidad EAFIT.

- Arévalo, P.; Ulloa, J. y Astudillo, S. (2008). Obtención de biodiesel a partir de grasa bovina. La granja, 8(2), 9-16.

- Atabani, A. E.; Silitonga, A. S.; Badruddina, I. A.; Mahliaa, T. M. I.; Masjukia, H. H. y Mekhilefd, S. (2012). A comprehensive review on biodiesel as an alternative energy resource and its characteristics. Renewable and Sustainable Energy Reviews, 16, 2070-2093.

- Awad, S.; Paraschiv, M.; Varuvel, E. G. y Tazerout, M. (2012). Optimization of Biodiesel production from animal fat residue in wastewater using response surface methodology. Bioresource Technology. http://dx.doi.org/10.1016/j.biortech.2012.11.086

- Balat, M. y Balat, H. (2010). Progress in biodiesel processing. Applied Energy, 87(6), 1815-35.

- Barrera, D. (2006). Evaluación de las propiedades de superficie del óxido de zinc y su actividad catalítica en la transesterificación del aceite de palma con metanol. Bogotá: Universidad de los Andes.

- Basha, S. A.; Gopal, K. R. y Jebaraj, S. (2009). A review on biodiesel production, combustion, emissions and performance. Renewable and Sustainable Energy Reviews, 13, 1628-1634.

- CEPAL -Comisión Económica para América Latina y el Caribe- y Naciones Unidas. (2007). Perspectivas para el biodiesel en Centroamérica: Costa Rica, El Salvador, Guatemala y Honduras. Lc/Mex/L.791.7.

- Darnoko, D. y Cheryan, M. (2000). Kinetics of palm oil transesterification in a batch reactor. JAOCS, 77(12), 1263-1267.

- Encinar, J. M.; Sánchez, N.; Martínez, G. y García, L. (2011). Study of biodiesel production from animal fats with high free fatty acid content. Bioresource Technology, 102, 10907-10914.

- Federación Nacional de Biocombustibles de Colombia. (2012). Cifras Informativas del Sector Biocombustibles biodiesel de palma de aceite. Recuperado el 12 de septiembre de 2012 de: http:www.fedebiocombustibles.com

- Fredman, B.; Butterfield, R. O. y Pryde, E. H. (1986). Tranesterification kinetics of Soybean. J. Am. Oil Chem. Soc., 63(10), 1375-1380.

- Keera, S. T.; El Sabagh, S. M. y Taman, A. R. (2011). Transesterification of vegetable oil to biodiesel fuel using alkaline catalyst. Fuel, 90, 42-47.

- Larosa, R. (2003). Proceso para la producción de BIODIESEL. Recuperado de 
http://www.zoetecnocampo.com/Documentos/biodie lar/biodie_lar.htm

- Lenoir, C. (2002). Análisis de la producción de Biodiesel. Recuperado de http://www.estrucplan.com.ar/Articulos/biodiesel.as p

- Lin, L.; Cunshan, Z.; Vittayapadung, S.; Xiangqian, S. y Mingdong D. (2011). Opportunities and challenges for biodiesel fuel. Applied Energy, 88(4), 1020-1031.

- Math, M. C.; Kumar, S. P. y Chetty, S.V. (2010). Technologies for biodiesel production from used cooking oil - A review. Energy for Sustainable Development, 14, 339-345.

- Oner, C. y Altun, S. (2009). Biodiesel production from inedible animal tallow and an experimental investigation of its use as alternative fuel in a direct injection diesel engine. Applied Energy, 86, 2114-2120.

- Rivera, I.; Villanueva, G. y Sandoval, G. (2009). Producción de biodiesel a partir de residuos grasos animales por vía enzimática. Grasas y aceites, 60(5), 468-474.

- Rojas, A. y Girón, E. (2011). Variables de operación en el proceso de transesterificación de grasas animales: una revisión. Universidad Nacional de Colombia, Palmira, Colombia.

- Shahid, E. M. y Jamal. J. (2011). Production of biodiesel: a technical review. Renew Sustain. Energy Rev, 15(9), 4732-45.

- Veira, J.; Grompone, M. y Jachmanián, I. (2004). Mejoramiento de las propiedades fisicoquímicas de la grasa vacuna para su uso en alimentos. Recuperado de http://www.buscagro.com/detailed/38780.html

- Vivas, A. (2010). Estudio y obtención de biodiesel a partir de residuos grasos de origen bovino. Pereira, Colombia: Universidad tecnológica de Pereira.

1. Magíster en Educación, Universidad del Norte. Candidata a Magíster en Ingeniería Ambiental, Universidad de Cartagena. Especialista en Química Analítica, Universidad de Cartagena. Ingeniera química, Universidad Industrial de Santander. Docente del grupo de investigación GIPIQ, línea de investigación en Tratamiento de Aguas Residuales Industriales (metales pesados). Docente de planta del programa de Ingeniería Química, Universidad de Cartagena, Colombia.candelariatejada@yahoo.com

2. Magíster en Ingeniería Ambiental, Universidad Nacional de Colombia. Candidata a Magíster en Ingeniería Mecánica, Universidad Tecnológica de Bolívar. Ingeniera Química, Universidad Industrial de Santander. Docente de planta del programa de Ingeniería Química, Universidad de Cartagena, Colombia. Iptbenitez@gmail.com 
3. Candidato a Magíster en Ingeniería Ambiental, Universidad de Cartagena. Especialista en Ingeniería Sanitaria y Ambiental, Universidad de Cartagena. Ingeniero Químico, Universidad Industrial de Santander. Docente de planta del programa de Ingeniería Química, Universidad de Cartagena, Colombia. angelvillabona@yahoo.es

4. Candidato a Magíster en Ciencia y Tecnología de Alimentos, Universidad de Cartagena. Especialista en Ciencia y Tecnología de Alimentos, Universidad Nacional. Ingeniero Químico, Universidad del Atlántico. Docente catedrático del programa de Ingeniería Química, Universidad de Cartagena, Colombia. Imonroyr@unicartagena.edu.co 